\title{
Effects of Kaolin particle size and annealing temperature on the Resistivity of Zinc- Kaolin Composite Resistors
}

\author{
BABALOLA, O.A.; AKOMOLAFE, T.
}

\author{
Physics Department, University of Ilorin, Nigeria.E-mail: babalolashadeyinka@yahoo.com 08052491009
}

\begin{abstract}
The effects of kaolin particle sizes on the resistivity of zinc-kaolin composite resistors have been investigated. The composite resistors have been produced from kaolin particle sizes ranging from $0.063 \mathrm{~mm}$ to 0.5 $\mathrm{mm}$. The resistors were produced in a mould to a dimension of $65 \mathrm{~mm} \times 6.5 \mathrm{~mm} \times 3.2 \mathrm{~mm}$ with dry zinc and kaolin powders to which a drop or two of sodium silicate have been added as a binder. The resistors were annealed at temperatures ranging from $300{ }^{\circ} \mathrm{C}$ to $1000{ }^{\circ} \mathrm{C}$ in steps of $100{ }^{\circ} \mathrm{C}$ for composites of $0 \%, 5 \%, 40 \%$ and $60 \%$ (vol.) zinc content. Results showed that composite resistivity at all the zinc concentrations considered and at all annealing temperatures increased exponentially with increasing kaolin particle size. The effect of annealing showed that composite resistance is highest for composites annealed at $1000{ }^{\circ} \mathrm{C}$ and lowest for cermets annealed between $500{ }^{\circ} \mathrm{C}$ and $600{ }^{\circ} \mathrm{C}$. Composite resistance increased with increasing kaolin particle size due to the large contact area presented by the insulating kaolin particles resulting in reduction of the number of zinc particle-particle contact. The results from this study will help in the development of conducting ceramics which could find use in chemical industries as electrodes in environments not conducive for metallic electrodes. @ JASEM
\end{abstract}

For most materials, an electric current results from the motion of free electrons, which are accelerated in response to an applied electric field. The number of these free electrons depends on the electron energy band structure of the material. The conduction mechanisms of cermets consistent with their microstructure have been studied by Nordsrom and Hills (1979) and by Prudenziati (1981). Electrical conductivity includes different possibilities i.e. hopping of electrons from and to conductive grains and localized states in the glassy matrix, direct tunnelling of electrons between near-neighbouring grains assisted by resonant centres in the intergranular material and according to Prudenziati (1981) conduction in narrow band of nearly delocalised states in the inter-granular material. Various investigators such as Wimmer et al (1974), Mizsei and Lantto(1991), Prudenziati et al (1991), Prudenziati and Acquab (1994), Akomolafe and Oladipo (1996), Afronte et al (1997) and Kuzy(1997) have sought to explain the factors contributing to the complex behaviours of cermet resistor systems. Researchers like Yoshikawa et al (1990) and Ruschau et al $(1992,1999)$ have worked on the effect of filler size on the resistivity of cermets. Percolation theory describes the resistivity change, which occurs when conducting fillers is loaded into a composite (Ruschau et al, 1990). When a certain critical volume fraction of the conductor is reached, the resistivity of the composite drops many orders of magnitude to a level more commensurate with the intrinsic filler resistivity (Zallen, 1983). However, all of these composites still have resistivities several orders of magnitude higher than the resistivities of the pure filler materials. The conductive spheres model developed by Hashin(1968) describes the conductivity of spheres with a thin insulating shell by the following relation

$$
\rho_{c}=\rho_{o x}\left[1+\frac{v_{m}}{\left[\left(\rho_{o x} / \rho_{m}\right)-1\right]^{-1}+\left(1-v_{m}\right) / 3}\right] 1 .
$$

where $\rho_{c}$ is the composite resistivity, $\rho_{o x}$ is the oxide resistivity, $v_{m}$ is the volume fraction of the metal and $\rho_{m}$ is the metal resistivity. Ruschau (1990) has explained this relation of the composite resistivity is inadequate because it is valid only for ohmic conduction; the oxide layer thicknesses are of the same order of magnitude as the electron tunnelling distances. Hence the resistivities of these composites are several orders of magnitudes lower than those that would be predicted by equation 1 . This relation also does not explain why composites with fillers that do not readily form oxides have resistivities several orders of magnitude higher than the metal resistivities even at loading as high as 90 $\%$ (vol.). The more plausible explanation is the effect of the particle-particle contact resistance. Because of the spherical nature of many processed powders, surface energy is minimised as the area of contact between particles is only a small fraction of the particle diameter (Ruschau, 1990). When a voltage is applied the resulting current is constricted to this small area; the resistance associated with this is known as the constriction resistance. In addition, any oxide (or other tarnish) layer prevents or at least limits good ohmic contacts between particles susceptible to oxidation. The resistance related to this film is known as the tunnelling resistance (Holms, 1967), also increases as the contact area decreases. Other than metal-polymer composites (Blaszkiwicz,

\footnotetext{
* Corresponding author: Babalola, O.A.
} 
1991 and Vilkacova, 2001), there are few articles, which investigate the grain boundary phenomenon in metal-ceramic composites. The purpose of this work is to study the variation of the resistivity of zinckaolin composites in relation to the clay particle size and its annealing temperatures.

\section{MATERIALS AND METHOD}

For the purpose of simplifying the experiments, the composite resistors fabricated were composed of only three components namely the zinc powder, which served as the conductive phase, kaolin powder which served as the insulating matrix and one or two drops of sodium silicate, which served as the binder. The resistors were all fabricated using a compaction method to compress carefully measured volume ratios of zinc and kaolin to a volume dimension $65 \mathrm{~mm} \times 6.6 \mathrm{~mm} \times 3.2 \mathrm{~mm}$ using a pressure of about $(6.35 \pm 0.02) \times 10^{8} \mathrm{~N} / \mathrm{m}^{2}$ in a rectangular mould. Different kaolin particle sizes were obtained using the standard sieve method; sieves of size $0.025 \mathrm{~mm}$, $0.063 \mathrm{~mm}, 0.10 \mathrm{~mm}, 0.20 \mathrm{~mm}, 0.30 \mathrm{~mm}, 0.40 \mathrm{~mm}$ and $0.50 \mathrm{~mm}$ were used. Each of the sieved kaolin particle sizes were used to fabricate composite resistors having $0,5,40$ and $60 \%$ (vol.) zinc content. These resistors were air-dried at room temperature for a duration of one week. The composite resistors were then heat-treated by firing them in an electric furnace at varying annealing temperatures $T_{\mathrm{f}}$ between $300{ }^{\circ} \mathrm{C}$ and $1000{ }^{\circ} \mathrm{C}$ in increasing steps of of $100{ }^{\circ} \mathrm{C}$ for a time duration of 60 minutes. Thereafter, the cermets were oven-cooled to room temperature to prevent them from developing internal stress and cracks, which could result from rapid temperature changes.

Measurement of DC resistivity was carried out using the two-probe technique at room temperature. Microstructural changes in the composite resistors were investigated using a Scanning Electron Microscope model Sterioscan 430i. The scanning electron microscope enabled characterisation of the resistors in terms of composition and microstructural evolution as the annealing temperatures increases. It also provides a means of estimating the average conducting particle size as the annealing temperature increases. X-ray Fluorescence (XRF) of the composite samples was also done using the Link Analytical (ISIS) using a maximum beam energy of $25 \mathrm{keV}$.

\section{RESULTS AND DISCUSSION}

The fraction/percentage composition of the insulating (kaolin) phase to the conductive (zinc filler) phase determines the overall bulk dc resistance and electrical properties of the zinc-kaolin cermets. The resistance of a cermet can be considered to be the result of a series of large number of resistor networks connected in series and in parallel with each particle and particle-particle contacts contributing to the bulk or total resistance. The three contributions to the bulk resistance of cermets are: constriction resistance associated with the constriction of the electron flow through the small area where any two conducting particles meet (Ruschau et al, 1992), tunnelling resistance associated with the insulating layer coating the conducting particles and the intrinsic grain resistance of each particle. The constriction resistance and the tunnelling resistance of the particle-particle contacts have been known to depend on the conducting particle size (Ruschau, 1972, Holm, 1967 and Ayodele and Akomolafe, 2005). We anticipated that changes in the insulating kaolin particle size would also affect the constriction resistance, the tunnelling resistance and hence the cermets bulk resistivity. Figures 1 and 2 shows the variation of cermets resistance with clay particle size for composites containing $5 \%$ (vol.) and $60 \%$ (vol.) zinckaolin ratio. It can be seen that cermets resistance increase exponentially with increasing kaolin particle size at all the annealing temperatures considered. This result is also consistent with those obtained for 0 $\%$ (vol.) and $40 \%$ (vol.) zinc content. 
$5 \%$ (vol. ) Zn-Clay Cermet

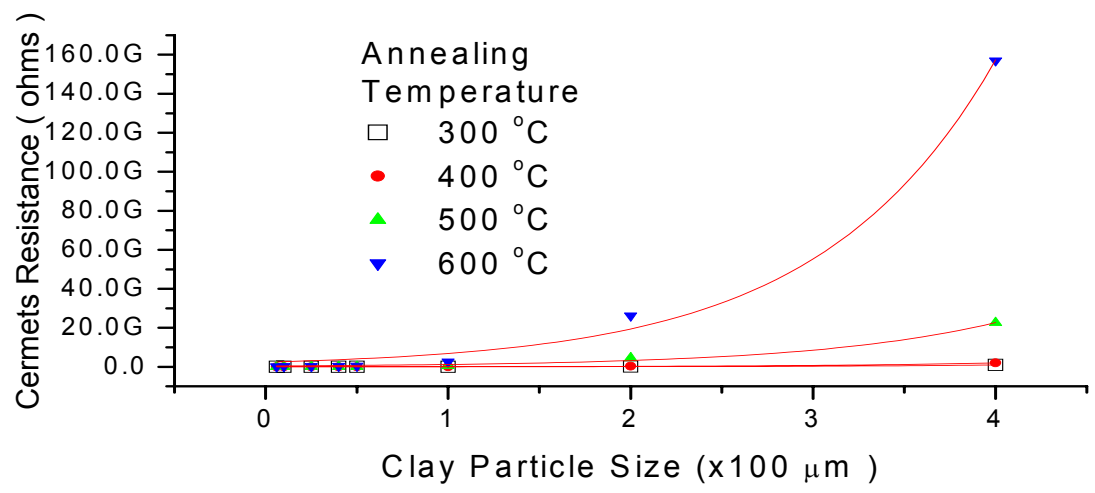

$5 \%$ (vol. ) Zn-Clay Cermet

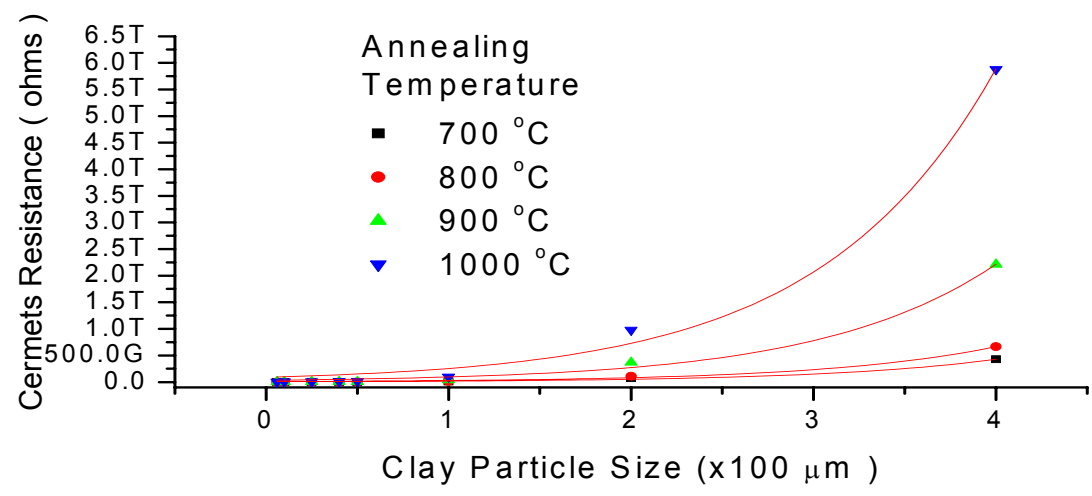

Fig. 1 Variation of Cermets Resistance with Clay Particle Size for $5 \%$ (vol.) Zn- Clay ratio. 

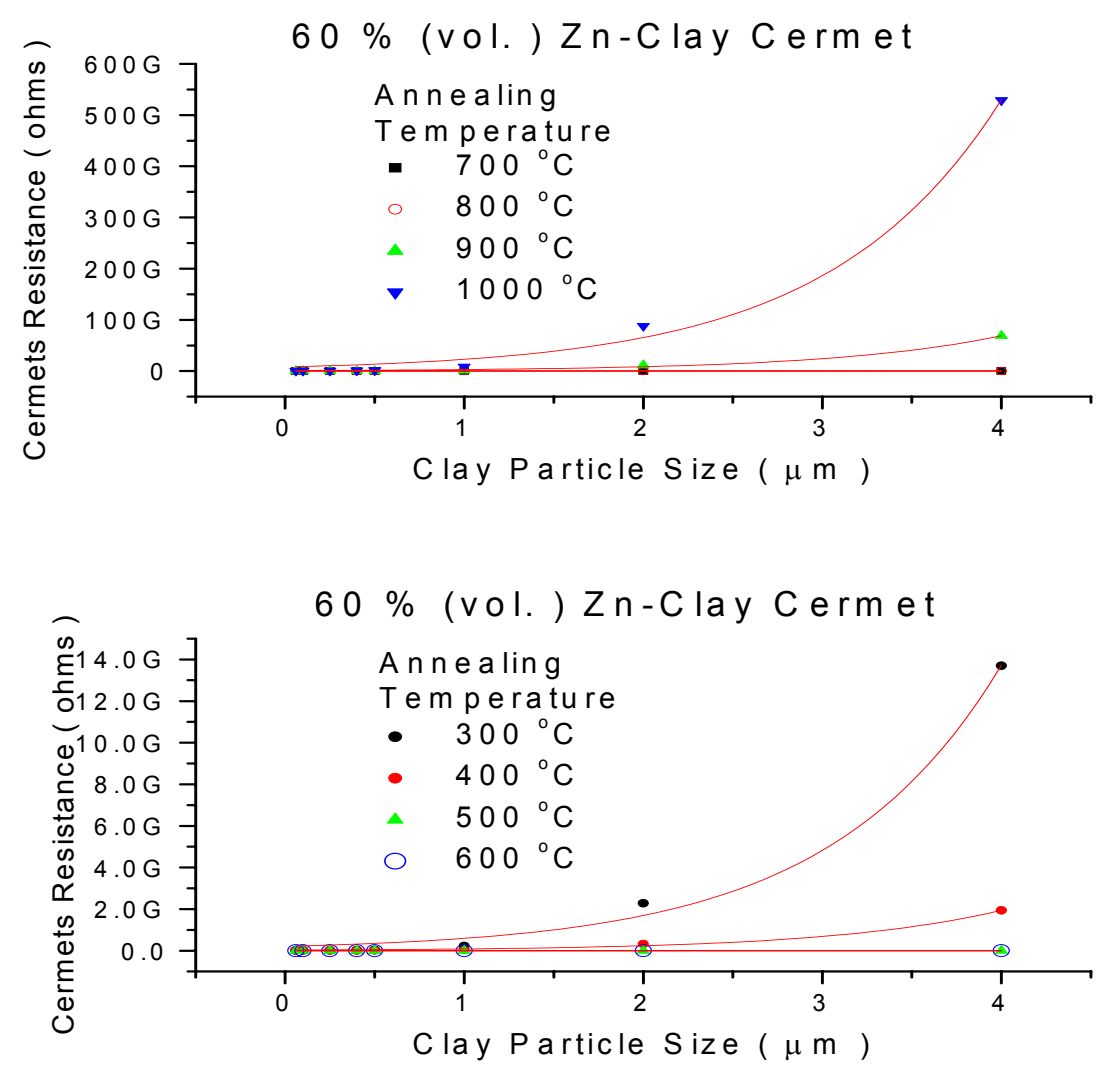

Fig. 2 Variation of Cermets Resistance with Clay Particle Size for 60\% (vol.) Zn- Clay ratio.

Smaller zinc particle results in more filler particleparticle contacts and interactions per conducting chain and larger contact areas, so that composites with small particle size have higher resistivities than composites with higher matrix particle size with equivalent volume loading. Factors responsible for the higher resistivities occurring for cermets, which possess higher kaolin matrix particle size, are the attendant reduction in compaction of metal-matrix particles. An increase in probability of micro-cracks existing in the cermets and increase in separation between zinc grains-leading to an increase in the electron tunnelling barrier width, which in turn increases the resistivity. Observation of the variation of resistance $\mathrm{R}$ with the kaolin particle diameter $\mathrm{d}$ at various concentrations has shown that the increase in resistance of the composite with kaolin particle size depend more on the on its particle size rather than the concentration of the filler since the variation of resistivity with matrix size increases exponentially in all cases. This shows that tunnelling resistance, which increases with increasing kaolin particle size, is the main contributor to the bulk resistance of the cermets. We estimated the activation energy $\mathrm{E}_{\mathrm{A}}$ of conduction by fitting the obtained variation of resistance with cermets temperature for temperatures between $40{ }^{\circ} \mathrm{C}$ and $120{ }^{\circ} \mathrm{C}$ with the Arrhenius-type equation

$$
R=R_{o} \exp \left(\frac{E_{A}}{k T}\right)
$$

where $\mathrm{T}$ is the cermets temperature, $\mathrm{k}$ is the Boltmznn's constant and Ro is the pre-exponential factor which depend on the mobility if the charge carriers (Vilkacova, 2001) for the $60 \%$ (vol.) zinckaolin composition at various concentrations from which we obtained the result in figure 3 which show the variation of activation energy of conductivity with kaolin particle size for the cermets. Results show that activation energy and hence tunnelling resistance increases with increasing kaolin particle size. 


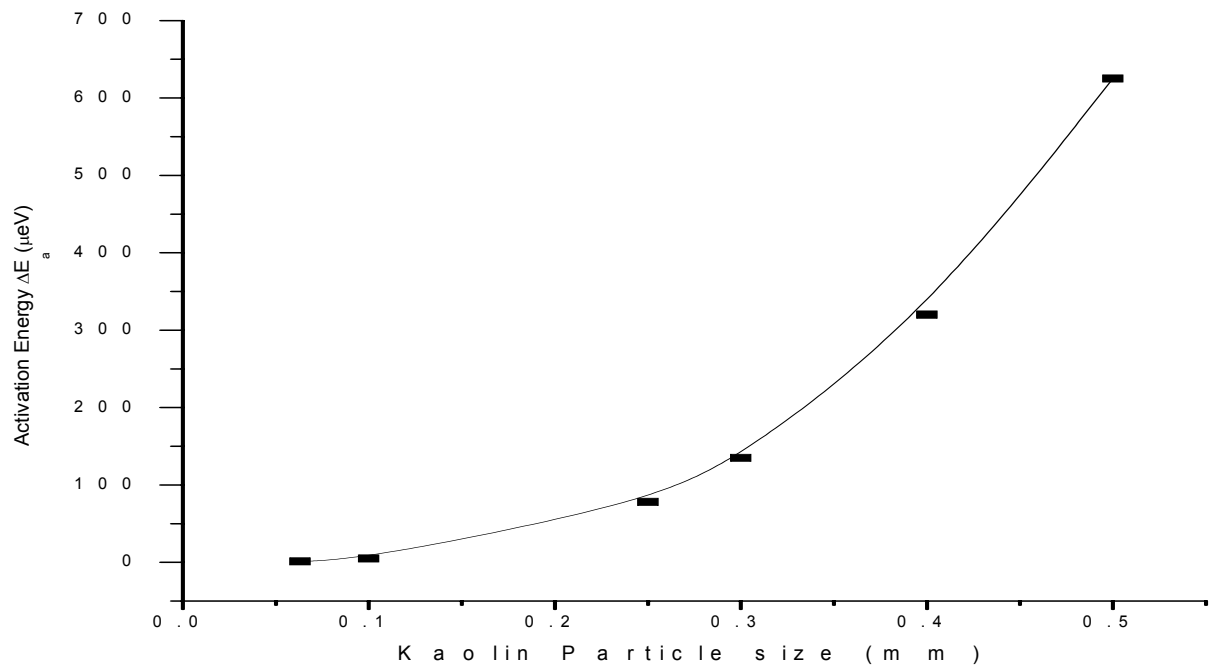

Fig. 3 Variation of Activation Energy with kaolin particle size for $60 \%$ (vol.) $\mathrm{Zn}$ composite resistors.

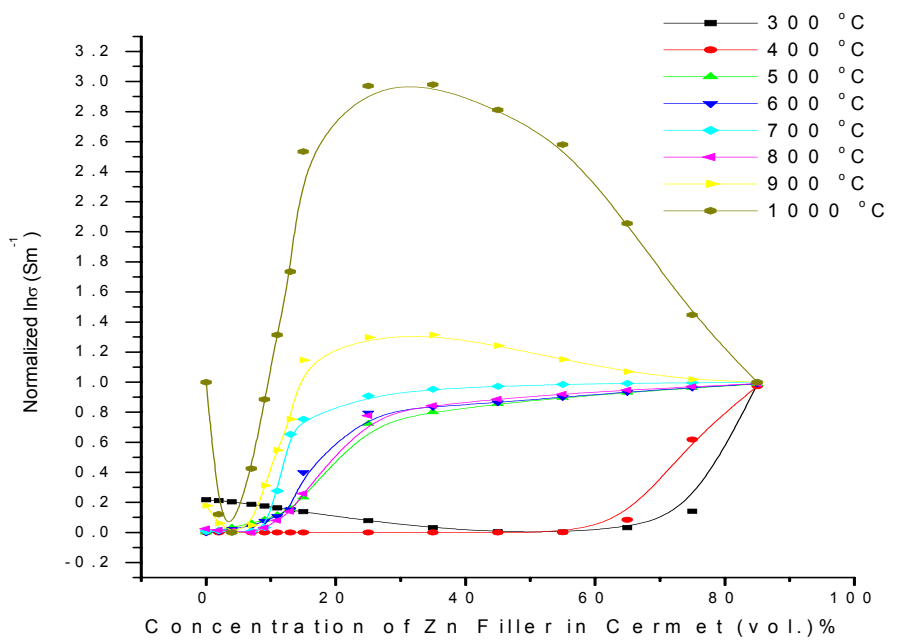

Fig. 4 Variation of Normalized $\ln \sigma$ with Filler concentration showing the variation of percolation threshold with annealing temperature

Figures 4 show the variation of the normalized natural $\log$ of conductivity with filler concentration. We observe that percolation threshold reduces with increasing annealing temperature. This shows that the grain size of the zinc particle size also increases as the annealing temperature increases from $400{ }^{\circ} \mathrm{C}$ to $600{ }^{\circ} \mathrm{C}$.

This increase in grain size (a result of melting and coagulation of molten zinc) as seen in figure 5, which shows the photomicrographs of zinc-kaolin cermet containing $70 \%$ (vol.) zinc and annealed at $500{ }^{\circ} \mathrm{C}$ reduced the effective/bulk resistivity of the cermets at these temperatures. Below a zinc filler concentration of $20 \%$ (vol.) however, the composite resistor is conducting below its percolation threshold so that its resistivity is very high at all annealing temperatures.

* Corresponding author: Babalola, O.A. 


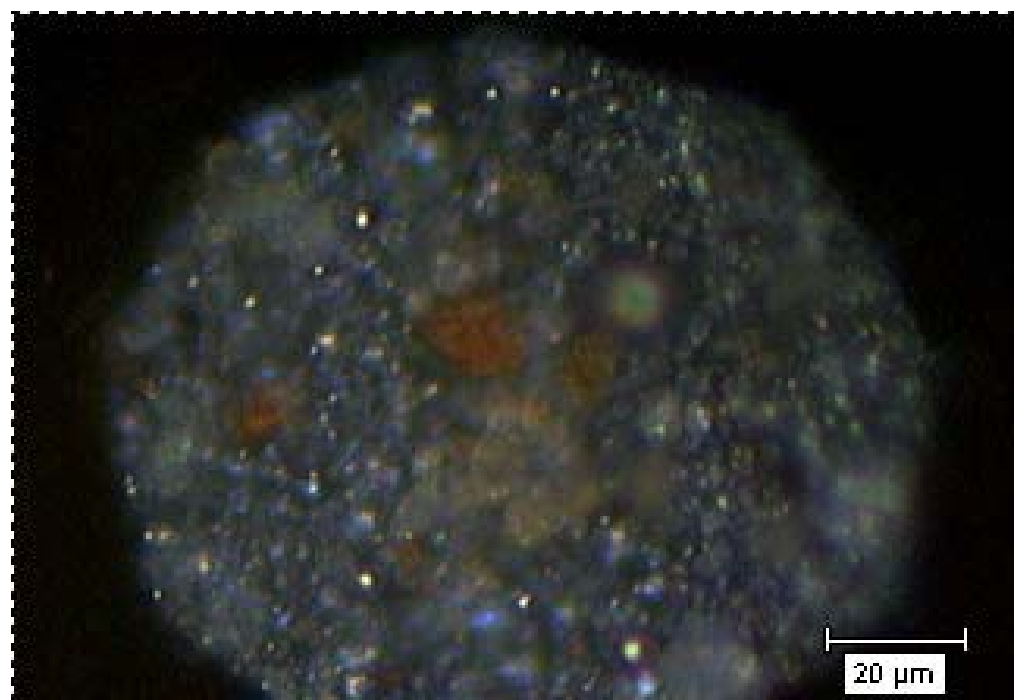

Fig. 5 Photomicrographs of Zn-clay Cermets containing $70 \%$ (vol.) Zinc annealed at 500

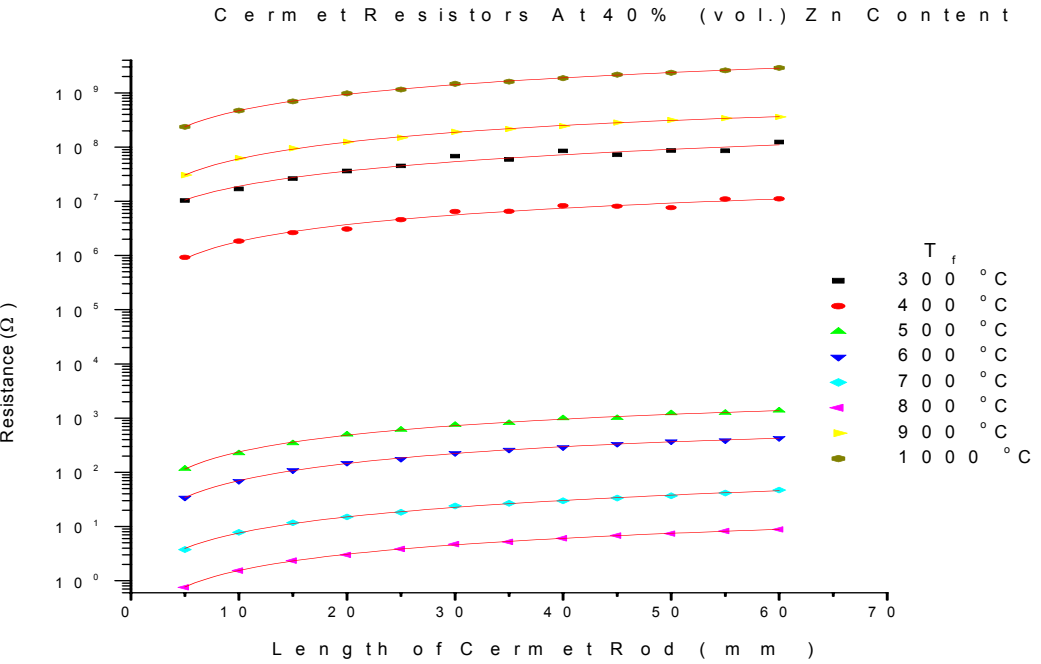

Fig. 6 Variation of Cermet resistance (emphasizing the effect of annealing Temperature) with resistor length for $\mathrm{Zn}$ concentration = $40 \%$ (vol.)Zn

Above a zinc filler concentration of $20 \%$ (vol.), the final resistivity of the cermet depend more on the annealing temperature. Figure 6 shows the variation of cermets resistance with resistor length, emphasizing the effects of annealing temperature $T_{f}$ on the cermets resistivities. We observe that resistivity of cermet is lowest for cermets annealed at temperatures between $500{ }^{\circ} \mathrm{C}$ and $800{ }^{\circ} \mathrm{C}$.

We propose that the sudden increase in resistivity of cermets annealed above $900{ }^{\circ} \mathrm{C}$ is due either to formation of complexes with the kaolin, oxidation or the possibility of vaporisation of the free zinc metal since its boiling point is about $900{ }^{\circ} \mathrm{C}$. The $\mathrm{x}$-ray fluorescence shown in figure 7 for the $70 \%$ (vol.) zinc content composite annealed at $1000{ }^{\circ} \mathrm{C}$ however shows that the zinc metal must have formed an insulating complex with the kaolinite atoms since it did not reveal a significant increase in oxygen

\footnotetext{
* Corresponding author: Babalola, O.A.
} 